\title{
MILTON'S CONJECTURE ON THE REGULARITY OF SOLUTIONS TO ISOTROPIC EQUATIONS
}

\author{
Daniel FARACO ${ }^{1}$ \\ Department of Mathematics, University of Jyväskylä, PO Box 35 (MaD), 40351 Jyväskylä, Finland
}

Received 19 April 2002, accepted 14 October 2002

ABSTRACT. - We present examples showing that the threshold for the integrability of the gradient of solutions to isotropic equations is $2 K /(K-1)$. The main tools are $p$-laminates and Beltrami Operators.

(C) 2003 L'Association Publications de l'Institut Henri Poincaré. Published by Elsevier B.V. All rights reserved

MSC: 30C62; 49J45; 35J15

RÉSUMÉ. - Nous présentons des exemples, qui prouvent que le seuil de l'intégrabilité du gradient des solutions des équations isotropiques est $2 K /(K-1)$. Les techniques principales sont les $p$-laminates et les opérateurs de Beltrami.

(C) 2003 L'Association Publications de l'Institut Henri Poincaré. Published by Elsevier B.V. All rights reserved

\section{Introduction}

In this paper we investigate the regularity of solutions to the isotropic equation

$$
\operatorname{div}(\rho(z) \nabla u(z))=0 \quad \text { in } Q,
$$

where $Q$ is a square in the plane $\mathbb{R}^{2}, u \in W^{1,2}(Q, \mathbb{R})$ and $\rho \in L^{\infty}(Q,[1 / K, K])$ is real valued. Through the whole paper $K$ is an arbitrary constant greater than one. In [24] Piccinini and Spagnolo proved that the solutions to (1.1) are locally Hölder continuous with exponent $4 / \pi \operatorname{Arctan}(1 / K)$. Isotropic equations belong to the class of linear elliptic equations,

$$
\operatorname{div}(\sigma(z) \nabla u(z))=0 \quad \text { in } Q,
$$

where $\sigma(z) \in \mathbf{M}^{2 \times 2}$ with $\sigma(z)=\sigma(z)^{t}$ and $1 / K|\xi|^{2} \leqslant\langle\xi, \sigma(z) \xi\rangle \leqslant K|\xi|^{2}$ for every $\xi \in \mathbb{R}^{2}$ and a.e. $z$ in $Q$. As in the isotropic case we require $u \in W^{1,2}(Q, \mathbb{R})$.

It goes back to Morrey [19] that the threshold for the Hölder regularity of the solution in the anisotropic case is only $1 / K$. Thus, in terms of Hölder continuity, solutions to

E-mail address: faraco@mis.mpg.de (D. Faraco).

${ }^{1}$ The author is supported by the Academy of Finland (Project \#53292). 
the isotropic equation are more regular that in the general case. On the other hand, an interesting result of Marino and Spagnolo states that isotropic equations are dense in the set of anisotropic equations with respect to the G-convergence, see [16].

The regularity of solutions to a PDE is also studied in terms of the integrability of the gradient. By the Sobolev Embedding Theorem if the gradient is integrable with exponent $p>2$, the function is Hölder continuous with exponent $1-2 / p$. A crucial phenomenon in elliptic PDE is that weak solutions which are a priory only in $W_{\text {loc }}^{1,2}(Q)$ automatically belong to $W_{\text {loc }}^{1, p}(Q)$ for some $p>2$ (see [8] for $n=2$ and [17] for arbitrary $n$ ). More precisely the gradients satisfy the so-called reverse Hölder inequalities, that is, for every ball $B(a, r)$ such that $B(a, 2 r)$ is compactly included in $Q$ there exists a constant $C(K, p)$ such that

$$
\int_{B(a, r)}|\nabla u|^{p} d z \leqslant C(K, p)\left(\int_{B(a, 2 r)}|\nabla u(z)|^{2} d z\right)^{p / 2} .
$$

In understanding the properties of a given subclass of elliptic PDE is important to find the supremum of those exponents $p$ for which gradients of weak solutions satisfy reverse Hölder inequalities. This supremum is called the threshold exponent of that class. The value of the threshold exponent is relevant in applications because it measures "the highest possible concentration of the field". See, for example, $[18,15]$ and the references therein for the relation of the threshold to several questions in physics.

The threshold for anisotropic equations like (1.2) in the plane was established by Astala, Leonetti and Nesi to be equal to $2 K /(K-1)$. The result was obtained by Leonetti and Nesi in [15] as a consequence of the higher integrability results for gradients of quasiregular mappings due to Astala [2], see also [10]. In the proofs in [2,10] the complex structure of $\mathbb{R}^{2}$ is essential and hence, the higher dimensional case remains as a challenging open problem. We see that in the anisotropic case the threshold for the integrability of the gradient $2 K /(K-1)$ and the Sobolev embedding yield the "right" Hölder regularity $1 / K$ (up to the end point). In fact, the example showing the sharpness of both results is the same: the real part of the radial stretching $f(z)=z|z|^{1 / K-1}$.

The search for the threshold for the integrability of the gradient in the isotropic case has also drawn the attention of the researchers, see for example [6] and [15]. A natural question is if also here the bounds for the Hölder continuity and the integrability of the gradient are related by the Sobolev embedding Theorem. In the related setting of quasiconformal mappings Pekka Koskela provided an example for which the Hölder regularity and the integrability of the gradient are not coupled by the Sobolev embedding [14].

The situation in the anisotropic case and the results in [24] indicate that the higher integrability threshold for the isotropic equations might be larger than $2 K /(K-1)$. However, the intuition coming from physics, led Graeme Milton to conjecture the opposite.

The underlying physical problem relies on the fact that the matrix valued function $\sigma$ in (1.2) can be thought of as to express the electric conductivity properties of certain material. In [18] Milton suggested conductivity matrices $\rho_{j} I$ where the concentration of the related fields $\nabla u_{j}$ should be high enough to prevent any uniform integrability better 
than $2 K /(K-1)$. However since his remarkable work appeared in 1986, a mathematical proof of this fact was lacking to the best of our knowledge. In this paper we rigorously prove Milton assertion showing that his physical intuition led him to the right answer. We present the result in the following form.

THEOREM 1.1. - Let $K>1$. There exist sequences of functions $\left\{\rho_{j}\right\} \in L^{\infty}(Q,\{K$, $1 / K\})$ and $\left\{u_{j}\right\} \in W^{1,2}(Q, \mathbb{R})$ with $\left\|u_{j}\right\|_{W^{1,2}} \leqslant 1$, such that

$$
\operatorname{div}\left(\rho_{j}(z) \nabla u_{j}(z)\right)=0 \quad \text { in } Q,
$$

and for every compact set $R$ of positive measure contained in $Q$

$$
\lim _{j \rightarrow \infty} \int_{R}\left|\nabla u_{j}(z)\right|^{2 K /(K-1)} d z=\infty .
$$

In fact our construction gives that the sequence $\left\{u_{j}\right\}$ is uniformly bounded in $W^{1, p}(Q, \mathbb{R})$ with $1 \leqslant p<2 K /(K-1)$. This must be the case, since the result of Astala-Leonetti-Nesi states that the bounds on the $W^{1,2}$ norm imply bounds on the $W^{1, p}$ norm for the above range of $p$.

Weak reverse Hölder inequalities imply also sharp regularity results for the Dirichlet problem

$$
\operatorname{div}(\sigma(z) \nabla u(z))=\operatorname{div} F \quad \text { in } Q,
$$

where $\sigma$ is as in (1.2) and $F \in L^{p}$ (see [12]). In this regard Theorem 1.1 is easily seen to imply the following corollary.

COROLLARY 1.2. - There exist functions $\rho \in L^{\infty}(Q,\{K, 1 / K\}), u \in W^{1,2}(Q, \mathbb{R})$ and a vector field $F \in L^{\infty}\left(Q, \mathbb{R}^{2}\right)$ such that

$$
\operatorname{div}(\rho(z) \nabla u(z))=\operatorname{div} F,
$$

and

$$
\int_{Q}|\nabla u(z)|^{2 K /(K-1)} d z=\infty .
$$

Our approach to study Eq. (1.1) is based upon considering the flow $\rho(z) \nabla u(z)$ as a rotated potential. Most of the notation used below is standard and explained in Section 2. However we need to introduce immediately the following sets. Let us associate to every positive number $\rho$ a 2-dimensional subspace $E_{\rho}$ of the space of $2 \times 2$ matrices $\mathbf{M}^{2 \times 2}$ as follows:

$$
E_{\rho}=\left\{\left(\begin{array}{c}
X \\
J \rho X
\end{array}\right) \text { where } X \in \mathbb{R}^{2} \text { and } J=\left(\begin{array}{ll}
0 & -1 \\
1 & 0
\end{array}\right)\right\}
$$

Denote

$$
E=E_{K} \cup E_{K^{-1}} .
$$


Then it can be seen that $u$ is a solution to a linear isotropic equation (1.1) with $\rho(z) \in\left\{K, K^{-1}\right\}$ almost everywhere if and only if $u$ is the real part of a function $f \in W^{1,2}\left(Q, \mathbb{R}^{2}\right)$ such that

$$
D f(z) \in E
$$

for almost every $z \in Q$. Moreover, set $k=(K-1) /(K+1)$. Then $D f(z) \in E$ if and only if there exists $\mu \in L^{\infty}(Q,\{-k, k\})$ such that

$$
\bar{\partial} f-\mu \overline{\partial f}=0 .
$$

The function $\mu$ is called the second complex dilatation of the mapping $f$ (see [1]). For the sake of completeness the relation between Eqs. (1.1) and (1.9) is discussed in Section 6.

Next we observe that the integrability properties of $D f$ are completely encoded in its distributional measure $D f_{\sharp} \mathcal{L}_{Q}^{n}$ (cf. Section 2), since

$$
\frac{1}{|Q|} \int_{Q}|D f(z)|^{p} d z=\int_{\mathbf{M}^{2 \times 2}}|\lambda|^{p} d D f_{\sharp}\left(\mathcal{L}_{Q}^{n}\right)(\lambda) .
$$

Thus our strategy will be the following: Firstly we construct a probability measure $v \in \mathcal{M}(E)$ such that

$$
\int_{\mathbf{M}^{2 \times 2}}|\lambda|^{2 K /(K-1)} d v(\lambda)=\infty .
$$

Since $v$ has support in $E$, if it was the distribution of the gradient of some Sobolev function the problem would be concluded. This need not to be the case, but using the theory of laminates (see Section 3 ) we can at least show the existence of a sequence $\left\{f_{j}\right\}$ uniformly bounded in each $W^{1, p}\left(Q, \mathbb{R}^{m}\right), 2 \leqslant p<2 K /(K-1)$ such that

$$
D f_{j_{\sharp}}\left(\mathcal{L}_{Q}^{n}\right) \stackrel{\star}{\rightarrow} v \quad \text { in } \mathcal{M}\left(\mathbf{M}^{2 \times 2}\right) .
$$

Whenever (1.10) holds we say that $\left\{D f_{j}\right\}$ generates the measure $v$. The last difficulty is that, a priory, the sequence $\left\{D f_{j}\right\}$ does not stay in $E$ almost everywhere. This can be handled by several means. One option is based in adapting the recent new methods for solving partial differential inclusions (see $[9,13,20]$ and in particular Proposition 4.42 in [13]) to our situation. However, the proof would be more technical and specific. We have chosen to follow a somehow more direct (familiar) and general route based on the so-called Beltrami Operators. Using them we can find another sequence $\left\{g_{j}\right\}$ such that $D g_{j}(z) \in E$ almost every $z \in Q$ and it also generates $v$. The latter argument is related to those of [5], where the Beltrami Operators were applied to analyze the socalled Quasiregular Gradient Young measures. These operators have turned out to be an efficient tool in clarifying a wealth of questions concerning the study of the best exponents in planar PDE and related topics $[3,11,5]$. Their invertibility properties and other issues are described in the recent work of Astala, Iwaniec and Saksman [4]. 
Once a sequence $\left\{g_{j}\right\}$ as above is obtained, an easy argument shows that $\left\{\operatorname{Re}\left(g_{j}\right)\right\}$ and the conductivity coefficients $\left\{\rho_{j}\right\}$ associated to the second complex dilatations of $g_{j}$ prove the Theorem 1.1 to be true.

\section{Notation}

Let $Q$ denote the $n$ dimensional unit cube $Q=\left\{z \in \mathbb{R}^{n}:\left|z_{i}\right| \leqslant 1\right\}, B(a, r)=\{z \in$ $\left.\mathbb{R}^{n}:|z-a| \leqslant r\right\}$ and $R \Subset Q$ means that $R$ is a compact subset of $Q$. Concerning matrices, $\mathbf{M}^{m \times n}$ is the space of $m \times n$ matrices. The tensor product $a \otimes d$ with $a \in \mathbb{R}^{m}$ and $d \in \mathbb{R}^{n}$ denotes the rank-one matrix $\left(a_{i} d_{j}\right)$. It maps $v \in \mathbb{R}^{n}$ to $\langle v, d\rangle a$. Here, $\langle\cdot, \cdot\rangle$ represents the Euclidean scalar product. Unless otherwise indicated for a matrix $A,|A|$ represents the Euclidean norm of $A$. We denote closed balls in the space of matrices $\mathbf{M}^{m \times n}$ by $B(r)$, i.e. $B(r)=\left\{A \in \mathbf{M}^{m \times n}:|A| \leqslant r\right\}$. Similarly, $B_{\infty}(r)=\{A \in$ $\left.\mathbf{M}^{m \times n}:|A| \geqslant r\right\}$. The plane of diagonal matrices in $\mathbf{M}^{2 \times 2}$ is denoted by $D$. We will use the notation

$$
\left(\begin{array}{cc}
d_{1} & 0 \\
0 & d_{2}
\end{array}\right)=\left(d_{1}, d_{2}\right) .
$$

For a matrix $A \in \mathbf{M}^{2 \times 2}$ we will also use complex coordinates $A=\left(A_{z}, A_{\bar{z}}\right)$. Here, $A_{z} \in$ $\mathbb{C}$ and $A_{\bar{z}} \in \mathbb{C}$ satisfy the following relation: Let us identify a vector $w=(x, y) \in \mathbb{R}^{2}$ with the complex number $w=x+i y$. Then it holds that for every vector $w \in \mathbb{R}^{2}$

$$
A w=A_{z} \cdot w+A_{\bar{z}} \cdot \bar{w},
$$

where $\bar{w}$ denotes the complex conjugate of $w$. Using this notation

$$
\begin{aligned}
& E_{K}=\left\{A=\left(A_{z}, A_{\bar{z}}\right) \in \mathbf{M}^{2 \times 2}: A_{\bar{z}}=k \overline{A_{z}}\right\} \quad \text { and } \\
& E_{1 / K}=\left\{A=\left(A_{z}, A_{\bar{z}}\right) \in \mathbf{M}^{2 \times 2}: A_{\bar{z}}=-k \overline{A_{z}}\right\},
\end{aligned}
$$

where the sets $E_{\rho}$ were introduced in (1.7) and $k=(K-1) /(K+1)$. We use also complex coordinates for the differential of a mapping $f \in W^{1, p}\left(\Omega, \mathbb{R}^{2}\right), \Omega \subset \mathbb{R}^{2}$;

$$
D f(z)=(\partial f(z), \bar{\partial} f(z)) .
$$

Concerning measures $\mathcal{M}\left(\mathbf{M}^{2 \times 2}\right)$ stands for the set of Radon measures in $\mathbf{M}^{2 \times 2}, \delta_{A}$ is a Dirac delta at $A, \operatorname{spt} v$ stands for the support of $v$ and $\stackrel{\star}{\rightarrow}$ means convergence in the weak star topology. For a set $E,|E|$ denotes its Lebesgue measure. Let $\Omega$ be a bounded measurable set. Then $\mathcal{L}_{\Omega}^{n}$ stands for the normalized Lebesgue measure restricted $\Omega$ so that $\mathcal{L}_{\Omega}^{n}(\Omega)=1$. Let $f$ be a measurable function $f: \Omega \rightarrow \mathbb{R}^{m}$ and $N$ a Borel set in $\mathbb{R}^{m}$. Then the push-forward $\mathcal{L}_{\Omega}^{n}$ under $f$ is given by

$$
f_{\sharp}\left(\mathcal{L}_{\Omega}^{n}\right)(N)=\mathcal{L}_{\Omega}^{n}\left(f^{-1}(N)\right) .
$$

We call $f_{\sharp}\left(\mathcal{L}_{\Omega}^{n}\right)$ the distribution measure of $f$. Finally the threshold $2 K /(K-1)$ is denoted by $p_{K}$. 


\section{Laminates}

In this section we describe a process to build probability measures which arise as weak star limits of distribution measures of gradients of Sobolev functions. The class of probability measures obtained by this process, named as laminates, were introduced in [22] to provide examples of the so-called Homogeneous Gradient Young measures. In the setting of homogenization, lamination of materials has been present from the very beginning, since it provides one of the few situations where the relation MicrostructureMacrostructure is relatively well understood. We recall the basics of Laminates, referring to $[13,21,23]$ for further details. The reader familiar with Gradient Young measures will recognize features of this theory in the discussion below.

Let us start with a matrix $A \in \mathbf{M}^{m \times n}$. Suppose that there exist matrices $B, C \in \mathbf{M}^{m \times n}$, a real parameter $\lambda \in[0,1]$ and vectors $a \in \mathbb{R}^{m}, d \in \mathbb{R}^{n}$ such that

$$
A=\lambda B+(1-\lambda) C \quad \text { and } \quad B-C=a \otimes d .
$$

Whenever (3.1) is satisfied we say that $B$ and $C$ are rank-one connected and that $[B, C]$ is a rank-one segment. Therefore using this jargon, $A$ is supposed to belong to certain rank-one segment.

Let $h$ be the saw-tooth function on the real line, obtained as the periodic extension of

$$
h(x)= \begin{cases}\lambda x & \text { if } 0 \leqslant x \leqslant 1-\lambda, \\ -(1-\lambda) x+(1-\lambda) & \text { if } 1-\lambda \leqslant x \leqslant 1 .\end{cases}
$$

We define $f(z)=A z-a h(\langle z, d\rangle)$ for $z$ in the unit cube $Q$. Clearly $f \in W^{1, \infty}\left(Q, \mathbb{R}^{m}\right)$ and $D f_{\sharp}\left(\mathcal{L}_{Q}^{n}\right)$ is equal to the measure $v=\lambda \delta_{B}+(1-\lambda) \delta_{C}$. To iterate the process it is convenient to consider the sequence $\left\{f_{j}\right\}_{j=1}^{\infty}, f_{j}(z)=1 / j f(j z)$. The reason is twofold. Firstly, while for each $j, D f_{j \sharp}\left(\mathcal{L}_{Q}^{n}\right)=v$ it also holds that if $R \Subset Q,|R|>0$

$$
D f_{j \sharp}\left(\mathcal{L}_{R}^{n}\right) \stackrel{\star}{\rightarrow} v .
$$

Secondly, if another sequence $\left\{\tilde{f}_{j}\right\}$ satisfies that $\lim _{j \rightarrow \infty}\left|\left\{z \in Q: D f_{j} \neq D \tilde{f}_{j}\right\}\right|=0$, $\left\{D \tilde{f}_{j}\right\}$ generates $v$ as well. This is very useful because by using cut-off functions ([23, Lemma 8.4]), or by using auxiliary matrices (for example [13, Lemma 3.2] and Fig. 1), we can find $\left\{\tilde{f}_{j}\right\}$ with fixed boundary values equal to $A z$ and such that for each $j$, $\left|\left\{z \in Q: D f_{j}(z) \neq D \tilde{f}_{j}(z)\right\}\right| \leqslant 1 / j$.

Thus, since in the construction we can replace the unit cube $Q$ by an arbitrary cube $\tilde{Q}$ we have obtained the following result.

Given an arbitrary cube $\tilde{Q} \subset \mathbb{R}^{n}$, there exists a sequence $\left\{\tilde{f}_{j}\right\}$ bounded in $W^{1, \infty}(\tilde{Q}$, $\mathbb{R}^{m}$ ) such that for every $\tilde{R} \Subset \tilde{Q},|\tilde{R}|>0$

$$
D \tilde{f}_{j_{\sharp}}\left(\mathcal{L}_{\tilde{R}}^{n}\right) \stackrel{\star}{\rightarrow} v
$$

and $\tilde{f}_{j}(z)-A z \in W_{0}^{1, \infty}\left(\tilde{Q}, \mathbb{R}^{m}\right)$. We say that we have splitted the measure $\delta_{A}$ as $\lambda \delta_{B}+(1-\lambda) \delta_{C}$. 


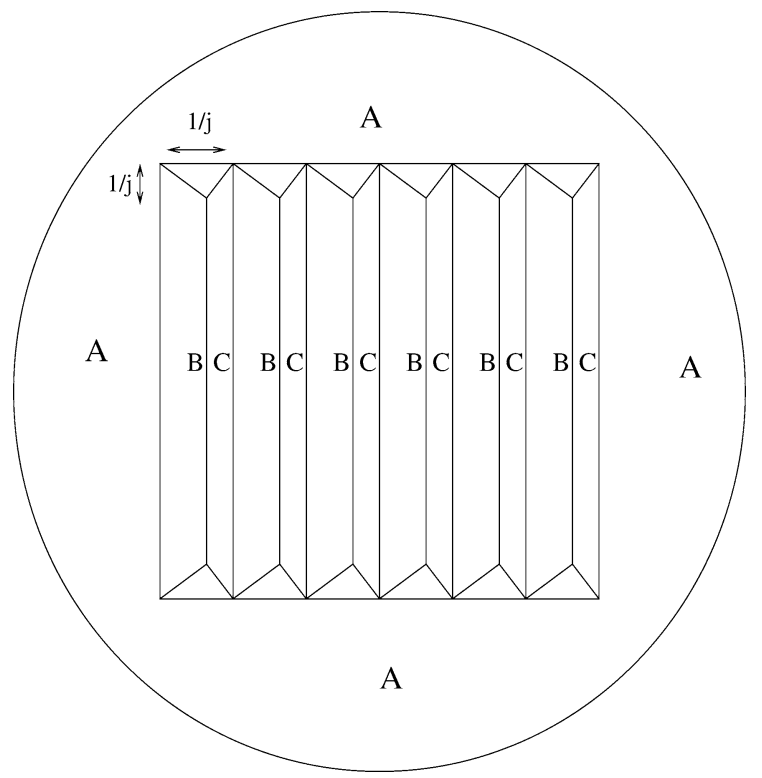

Fig. 1. If a piecewise affine mapping $f$ has gradient equal to $A$ in a certain region and $A$ belongs to a rank-one segment $[B, C]$, we can replace $A$ by layers where the gradient is equal to $B$ and $C$ and an interface region. The size of the interface is controlled by the number of layers we take.

Next, suppose that $B$ belongs to a rank-one segment $[D, E], B=\lambda_{2} D+\left(1-\lambda_{2}\right) E$, $0<\lambda_{2}<1$. We want to show that $v^{2}=\lambda\left(\lambda_{2} \delta_{D}+\left(1-\lambda_{2}\right) \delta_{E}\right)+(1-\lambda) \delta_{C}$ is also generated by gradients.

To obtain a sequence $\left\{f_{j}^{2}\right\}$ generating $v^{2}$ the idea is to modify the original sequence $\left\{f_{j}\right\}$, which generates $v$, on the set $\Omega_{j}=\left\{z \in Q: D f_{j}(z)=B\right\}$. The gradients of $f_{j}^{2}$ will take essentially values $D$ and $E$ on $\Omega_{j}$ and $A$ on $\mathbb{D} \backslash \Omega_{j}$. In fact, an auxiliar region where $D f_{j}^{2} \notin\{A, D, E\}$ is needed but its measure will converge to zero.

Formally, we take a finite collection of dyadic cubes $\left\{Q_{j}^{i}\right\}_{i=1}^{N_{j}} \subset \Omega_{j}$ such that $\mid \Omega_{j} \backslash$ $\bigcup_{i}^{N_{j}} Q_{j}^{i} \mid \leqslant 1 / j$. Inside of each of the cubes $Q_{j}^{i}$ we construct the sequence $\left\{f_{j, i}^{k}\right\}_{k=1}^{\infty} \in$ $W^{1, \infty}\left(Q_{j}^{i}, \mathbb{R}^{m}\right)$ obtained as in (3.2) but replacing $A$ by $B, v$ by $\lambda_{2} \delta_{D}+\left(1-\lambda_{2}\right) \delta_{E}$ and $\tilde{Q}$ by $Q_{j}^{i}$. We choose $k(j)=j N_{j}$ to have that $\left|\bigcup_{i=1}^{N_{j}}\left\{z \in Q_{j}^{i}: D f_{j, i}^{k(j)} \neq\{D, E\}\right\}\right| \leqslant 1 / j$.

Due to the affine boundary values of each $f_{j, i}^{k}$ we can weld them together and define

$$
f_{j}^{2}(z)= \begin{cases}f_{j, i}^{k(j)}(z) & \text { if } z \in Q_{j}^{i} \\ f_{j}(z) & \text { otherwise }\end{cases}
$$

A direct computation shows that for every $R \Subset Q,|R|>0$ the weak star limit of the sequence of measures $\left\{D f_{j_{\sharp}}^{2}\left(\mathcal{L}_{R}^{n}\right)\right\}$ is $v^{2}=\lambda\left(\lambda_{2} \delta_{D}+\left(1-\lambda_{2}\right) \delta_{E}\right)+(1-\lambda) \delta_{C}$. Clearly, we can iterate this construction as long as we have enough relations in term of rankone connections. The obtained measures will be generated by gradients in the sense of (1.10). We arrive at the class of prelaminates. 
DEFINITION 3.1. - The family of prelaminates $\mathcal{P} \mathcal{L}$ is the smallest family of probability measures on $\mathbf{M}^{m \times n}$ such that

(1) $\mathcal{P} \mathcal{L}$ contains all Dirac masses in $\mathbf{M}^{m \times n}$.

(2) Let $v=\sum_{i=1}^{k} \lambda_{i} \delta_{A_{i}} \in \mathcal{P} \mathcal{L}$ and let $A_{1}=\lambda B+(1-\lambda) C$ where $\lambda \in[0,1]$ and $[B, C]$ is a rank-one segment. Then the probability measure $\sum_{i=2}^{k} \lambda_{i} \delta_{A_{i}}+$ $\lambda_{1}\left(\lambda \delta_{B}+(1-\lambda) \delta_{C}\right) \in \mathcal{P} \mathcal{L}$.

THEOREM 3.2. - Let $v$ be a prelaminate supported in the ball $B(r) \subset \mathbf{M}^{m \times n}$. Then there exists a sequence $\left\{f_{j}\right\} \in W^{1, \infty}\left(Q, \mathbb{R}^{m}\right)$ such that for every $R \Subset Q,|R|>0$,

(1) $\left\|f_{j}\right\|_{1, \infty} \leqslant C r$,

(2) $D f_{j_{\sharp}}\left(\mathcal{L}_{R}^{n}\right) \stackrel{\star}{\rightarrow} v$.

Proof. - This theorem can be found in many places in the literature since it follows from the fact that laminates are homogeneous Gradient Young measures, [22]. The interested reader can complete a proof using the above scheme and an induction argument.

Finally laminates are defined as weak $(\star)$ limits of prelaminates in $\mathcal{M}\left(\mathbf{M}^{m \times n}\right)$. A laminate which is not a prelaminate is called an infinite-rank laminate.

Definition 3.3. - Let $v$ be a probability measure on $\mathbf{M}^{m \times n}$ and $1 \leqslant p<\infty$. Then $v$ is said to be a p-laminate if there exists a sequence of prelaminates $v_{j}$ such that

(a) $\sup _{j} \int_{\mathbf{M}^{m \times n}}|\lambda|^{p} d v_{j}(\lambda)<\infty$,

(b) $v_{j} \stackrel{\star}{\rightarrow} v$ in $\mathcal{M}\left(\mathbf{M}^{m \times n}\right)$.

THEOREM 3.4. - Let $v$ a p-laminate. Then there exists a sequence $\left\{f_{j}\right\}$ uniformly bounded in $W^{1, p}\left(Q, \mathbf{R}^{2}\right)$ such that

$$
D f_{j_{\sharp}}\left(\mathcal{L}_{R}^{n}\right) \stackrel{\star}{\rightarrow} v
$$

for every compact subset $R$ of $Q$ with positive measure.

Proof. - In the case of compactly supported laminates this theorem is proved in the literature (see [23], Chapter 9). The proof follows from Theorem 3.2 and a diagonalization argument. For the case of finite $p$ let us apply Theorem 3.2 to each prelaminate $v_{j}$. For each $j$ we obtain a sequence $\left\{f_{j}^{i}\right\}_{i=1}^{\infty}$ uniformly bounded in $W^{1, \infty}\left(Q, \mathbb{R}^{m}\right)$ such that $D f_{j_{\sharp}}^{i}\left(\mathcal{L}_{R}^{n}\right) \stackrel{\star}{\rightarrow} v_{j}$ as $i$ tends to $\infty$. The uniform bound on the $W^{1, \infty}$ norms of the $f_{j}^{i}$ gives that

$$
\lim _{i \rightarrow \infty} \int_{Q}\left|D f_{j}^{i}(x)\right|^{p} d x=\int_{\mathbf{M}^{m \times n}}|\lambda|^{p} d v_{j}(\lambda) .
$$

Putting this together with the assumption (a) in the definition of $p$ laminate gives the uniform bounds for the $p$-norms of the $\left\{f_{j}^{i}\right\}_{i, j=1}^{\infty}$ and thus for the generating subsequence.

The following remark, on the particular nature of the laminate we are going to deal with, will simplify the proofs in Section 5. 
Remark 3.5. - It is easy to see that if a $p$-laminate $v$ is purely atomic and

$$
\lim _{j \rightarrow \infty} v\left(\operatorname{spt}(v) \backslash \operatorname{spt}\left(v_{j}\right)\right)=0,
$$

$v_{j}$ as in Definition 3.3(b), the sequence $\left\{f_{j}\right\}$ obtained from Theorem 3.4 satisfies the following property: Let $\Omega_{j}=\left\{z \in Q: D f_{j}(z) \notin \operatorname{spt}(v)\right\}$. Then,

$$
\lim _{j \rightarrow \infty}\left|\Omega_{j}\right|=0 .
$$

\section{The staircase-laminate}

This section will be devoted to constructing a laminate $v$ supported in the set $E$ presented in formula (1.8), and satisfying

$$
\int_{\mathbf{M}^{2 \times 2}}|\lambda|^{p_{K}} d v(\lambda)=\infty \text { and } \int_{\mathbf{M}^{2 \times 2}}|\lambda|^{p} d \nu(\lambda)<\infty
$$

for every $p<p_{K}$. In fact, we will not need the whole set $E$ since the laminate $v$ will live on the intersection of $E$ with the plane of all diagonal matrices $D=\left(d_{1}, d_{2}\right)$; c.f. (2.1). Recall that in this plane the only rank-one directions are horizontal and vertical lines. Moreover, using notation (2.1), we have that

$$
E_{K} \cap D=\{(a, K a): a \in \mathbb{R}\} \quad \text { and } \quad E_{K^{-1}} \cap D=\left\{\left(a, K^{-1} a\right): a \in \mathbb{R}\right\} .
$$

It turns out that it is the opening of the cone $\mathcal{Q}$,

$$
\mathcal{Q} \equiv\left\{(x, y) \in D: K^{-1} \leqslant \frac{y}{x} \leqslant K\right\},
$$

what determines if it is possible to find a laminate $v$ supported in $Q$ such that $v\left(B_{\infty}(R)\right)$ converges to 0 slowly enough for (4.1) to hold.

We will firstly describe how certain sequences of matrices in $\mathcal{Q}$ give naturally rise to infinite rank-laminates and after that we will choose an appropriate sequence to create the measure $v$.

Our construction will resemble an staircase (see Fig. 3). Thus, we start by describing how to build its steps. Take two diagonal matrices $A_{1}=\left(A_{1}^{1}, A_{1}^{2}\right), A_{2}=\left(A_{2}^{1}, A_{2}^{2}\right) \in \mathcal{Q}$. We will use the partial ordering,

$$
A_{1} \leqslant A_{2} \quad \Leftrightarrow \quad A_{1}^{1} \leqslant A_{2}^{1} \text { and } A_{1}^{2} \leqslant A_{2}^{2} .
$$

Given such a pair of matrices, $B=\left(A_{1}^{1}, 1 / K A_{1}^{1}\right) \in E_{K^{-1}}, D=\left(A_{1}^{1}, A_{2}^{2}\right)$ and $C=$ $\left(A_{2}^{2} / K, A_{2}^{2}\right) \in E_{K}$ satisfy that $A_{1} \in[B, D], D \in\left[C, A_{2}\right]$. In addition, $[B, D]$ is a vertical segment and $\left[C, A_{2}\right]$ is an horizontal segment i.e. they are rank-one segments. For a quick illustration see Fig. 2 (observe that although in the figure $A_{1}^{1}=A_{2}^{2}$, this is not 


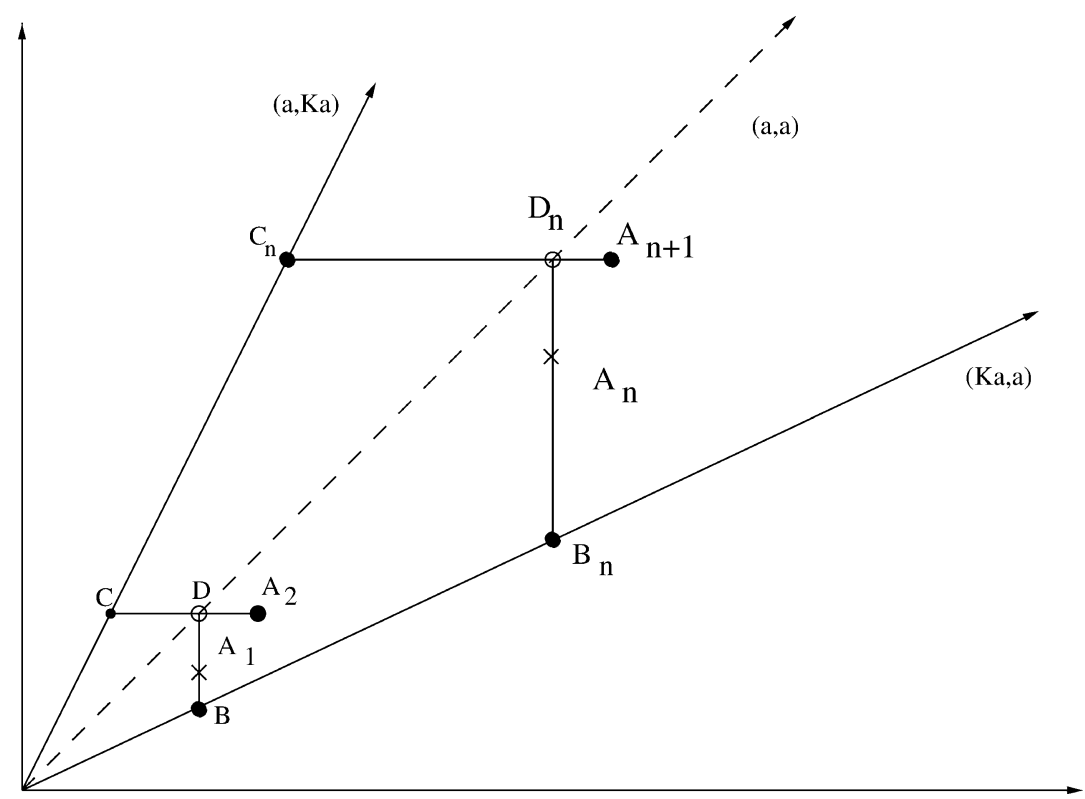

Fig. 2. "Steps" 1 and $n$. Black dots denote the support of the measure, the cross is the centre of mass, and white dots are auxiliary matrices.

required in the general construction). Let $\lambda_{1}$ and $\lambda_{2} \in[0,1]$ be parameters such that

$$
A_{1}=\lambda_{1} B+\left(1-\lambda_{1}\right) D \quad \text { and } \quad D=\lambda_{2} C+\left(1-\lambda_{2}\right) A_{2} .
$$

Plugging the latter expression into the former we obtain that

$$
A_{1}=\lambda_{1} B+\left(1-\lambda_{1}\right)\left(\lambda_{2} C+\left(1-\lambda_{2}\right) A_{2}\right) .
$$

In the language of measures (4.3) means

$$
A_{1}=\int_{\mathbf{M}^{2 \times 2}} t d v_{1}(t),
$$

where $v_{1}$ is the measure

$$
\nu_{1}=\lambda_{1} \delta_{B}+\left(1-\lambda_{1}\right)\left(\lambda_{2} \delta_{C}+\left(1-\lambda_{2}\right) \delta_{A_{2}}\right) .
$$

Fig. 2 shows how this construction looks like if the matrices are near the origin $\left(A_{1}\right.$ as the center of mass of a measure supported on $B, C$ and $A_{2}$ ), or if they are relatively far away ( $A_{n}$ as the center of mass of a measure supported on $B_{n}, C_{n}$ and $\left.A_{n+1}\right)$.

Now let us suppose that we are given a sequence of matrices $\left\{A_{n}\right\}_{n=1}^{\infty} \in \mathcal{Q}$ ordered as in (4.2), $A_{n} \leqslant A_{n+1}$ for every $n$. We can repeat the explained construction with $A_{1}=A_{n}$ and $A_{2}=A_{n+1}$. This yields a sequence of step measures $\left\{v_{n}\right\}_{n=1}^{\infty}$. We would like to paste the measures $v_{n}$ together to obtain a new measure $v$. Let us sketch the idea. Consider 


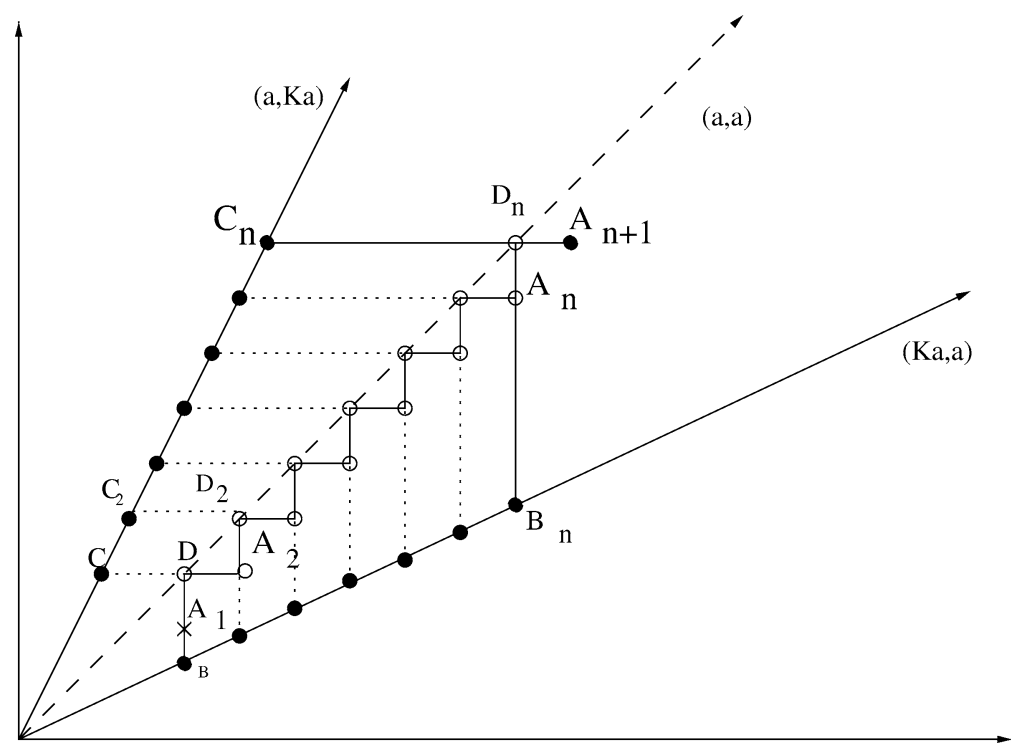

Fig. 3. The staircase at level $n$.

the measure $v_{1}$ as in (4.4). Replace $\delta_{A_{2}}$ in the definition of $v_{1}$ by the measure $v_{2}$. This defines a new measure

$$
v^{2}=\lambda_{1} \delta_{B}+\left(1-\lambda_{1}\right)\left(\lambda_{2} \delta_{C}+\left(1-\lambda_{2}\right)\left(\lambda_{3} \delta_{B_{2}}+\left(1-\lambda_{3}\right)\left(\lambda_{4} \delta_{C_{2}}+\left(1-\lambda_{4}\right) \delta_{A_{3}}\right)\right)\right) .
$$

Here the new parameters and matrices come from the definition of the step measure $v_{2}$. Since $v^{2}$ has an atom $\delta_{A_{3}}$, we proceed by replacing it by the step measure $v_{3}$ to obtain a new measure $v^{3}$ with an atom at $A_{4}$. We continue iteratively obtaining a sequence of probability measures $\left\{v^{n}\right\}$. Finally the measure $v$ is defined as the weak star limit of this sequence. Besides the condition on the ordering (4.2), the only restriction on the sequence $\left\{A_{n}\right\}$ is that $v$ should have finite $p$ th-moment for some $1<p<\infty$. We further observe that we have only used rank-one segments at every step of the construction, so it follows that if $v$ has finite $p$ th moment for some $p>1, v$ is a laminate. Fig. 3 and the concrete example below should help to understand the process just loosely explained.

Let us concentrate now in obtaining the measure $v$ such that (4.1) holds. We consider the sequence $A_{n}=\{(n+1, n)\}_{n=n_{0}}^{\infty}, n_{0} \geqslant 1 /(K-1)$. Since $\left\{A_{n}\right\}$ is well ordered and contained in $\mathcal{Q}$ we can use the scheme indicated above to construct prelaminates $v^{n}$ with centre of mass $A_{n_{0}}$, and supported on the set $E \cup\left\{A_{n+1}\right\}$. To avoid keeping track of $n_{0}$ everywhere we assume without loss of generality that $n_{0}=1$.

Let start with the measure $\delta_{A_{1}}$. Clearly the following relations hold,

$$
\begin{aligned}
& (2,1)=\frac{K}{2(K-1)}\left(2, \frac{2}{K}\right)+\left(1-\frac{K}{2(K-1)}\right)(2,2), \\
& (2,2)=\frac{K}{2(K-1)+K}\left(\frac{2}{K}, 2\right)+\left(1-\frac{K}{2(K-1)+K}\right)(3,2) .
\end{aligned}
$$


Thus, in the above notation, $B=(2,2 / K), D=(2,2)$ and $C=(2 / K, 2)$. Hence $A_{1}$ is the centre of mass of the probability measure $v_{1}$ defined by

$$
\begin{aligned}
v_{1}= & \frac{K}{2(K-1)} \delta_{(2,2 / K)}+\left(1-\frac{K}{2(K-1)}\right)\left(\frac{K}{2(K-1)+K} \delta_{(2 / K, 2)}\right. \\
& \left.+\left(1-\frac{K}{2(K-1)+K}\right) \delta_{(3,2)}\right),
\end{aligned}
$$

which is a prelaminate. Furthermore it can be expressed as $v_{1}=\mu_{1}+\lambda_{1} \delta_{A_{2}}$, where $\mu_{1}$ is a new measure supported in the set $E \cap B\left(2 C_{K}\right)$. The constant $C_{K}$ is equal to $|B|=|C|$, explicitly $C_{K}=|(1,1 / K)|=\sqrt{1+K^{2}} / K$. It will appear often below since it is a natural parameter in our construction. We say that we are one "step" up in the staircase.

The construction gives that $v_{1}\left(B_{\infty}\left(2 C_{K}\right)\right)=v_{1}\left(A_{2}\right)=\lambda_{1}$ where

$$
\lambda_{1}=\left(1-\frac{K}{2(K-1)+K}\right)\left(1-\frac{K}{2(K-1)}\right) .
$$

We can repeat the same operation at level $n$ since,

$$
\begin{aligned}
(n+1, n)= & \frac{K}{(n+1)(K-1)}\left(n+1, \frac{n+1}{K}\right) \\
& +\left(1-\frac{K}{(n+1)(K-1)}\right)(n+1, n+1), \\
(n+1, n+1)= & \frac{K}{(n+1)(K-1)+K}\left(\frac{n+1}{K}, n+1\right) \\
& +\left(1-\frac{K}{(n+1)(K-1)+K}\right)(n+2, n+1) .
\end{aligned}
$$

The structure relations in terms of rank-one connections are the same as in (4.5). Hence $A_{n}$ can be expressed as the center of mass of the laminate $v_{n}$ defined by

$$
\begin{aligned}
v_{n}= & \frac{K}{(n+1)(K-1)} \delta_{(n+1,(n+1) / K)} \\
& +\left(1-\frac{K}{(n+1)(K-1)}\right)\left(\frac{K}{(n+1)(K-1)+K} \delta_{((n+1) / K, n+1)}\right. \\
& \left.+\left(1-\frac{K}{(n+1)(K-1)+K}\right) \delta_{(n+2, n+1)}\right) .
\end{aligned}
$$

As before there exists a measure $\mu_{n}$ supported in $E \cap B\left((n+1) C_{K}\right)$ such that $v_{n}$ splits as $v_{n}=\mu_{n}+\lambda_{n} \delta_{A_{n+1}}$ and $v_{n}\left(B_{\infty}\left((n+1) C_{K}\right)\right)=\lambda_{n}$. The value of $\lambda_{n}$ will be important:

$$
\lambda_{n}=\left(1-\frac{K}{(n+1)(K-1)+K}\right)\left(1-\frac{K}{(n+1)(K-1)}\right) .
$$

Next, we paste the steps together to obtain a truncated staircase. The formal procedure is done by induction. Let us start with $n=1$. Remember that

$$
v_{1}=\mu_{1}+\lambda_{1} \delta_{A_{2}} .
$$


We define

$$
v^{2}=\mu_{1}+\lambda_{1} v_{2}
$$

Declare $\mu^{2}=v_{\mid B\left(3 C_{K}\right)}^{2}$. Then $v^{2}$ splits in the form $v^{2}=\mu^{2}+\lambda_{1} \lambda_{2} \delta_{A_{3}}$ and $\mu^{2}$ is supported in $E \cap B\left(3 C_{k}\right)$. Since $A_{3}$ is the center of mass of $\nu_{3}$, we defined $\nu^{3}=\mu^{2}+\lambda_{1} \lambda_{2} \nu_{3}$. Now we can find $\mu^{3}$ as before and continue inductively. The previous procedure gives the definitions:

$$
v^{n}=\mu^{n-1}+\left(\prod_{i=1}^{n-1} \lambda_{i}\right) v_{n}
$$

and

$$
\mu^{n}=v_{\mid B\left((n+1) C_{K}\right)}^{n} .
$$

This defines the truncated staircases. Observe that it follows from the construction that

$$
v^{m}\left(B_{\infty}\left((n+1) C_{K}\right)\right)=v^{n}\left(B_{\infty}\left((n+1) C_{K}\right)\right)=v^{n}\left(A_{n+1}\right)=\prod_{i=1}^{n} \lambda_{i}
$$

for every $m \geqslant n$. Finally, we let the staircase grow infinitely and obtain:

DEFINITION 4.1 (The staircase-laminate). - Let $v^{n}$ be as above. Then the staircaselaminate $v$ is defined by:

$$
v=\lim _{n \rightarrow \infty} v^{n} \quad \text { in the weak star topology of } \mathcal{M}\left(\mathbf{M}^{2 \times 2}\right) .
$$

Now we need to guarantee that $v$ is a probability measure with the appropriated growth.

First we observe that by the Cavalieri principle,

$$
\int_{\mathbf{M}^{2 \times 2}}|\lambda|^{p} d v(\lambda)=p \int_{0}^{\infty} t^{p-1} v\left(B_{\infty}(t)\right) d t
$$

Next we let $m$ go to infinity in (4.8) to obtain that

$$
v\left(B_{\infty}\left((n+1) C_{K}\right)\right)=v^{n}\left(A_{n+1}\right)=\prod_{i=1}^{n} \lambda_{i} .
$$

By inserting (4.11) into (4.10) it is easy to see that,

$$
\int_{\mathbf{M}^{2 \times 2}}|\lambda|^{p} d v(\lambda) \approx \sum_{n=1}^{\infty} n^{p-1} v^{n}\left(A_{n+1}\right) .
$$

We compare the above sum with $\sum_{n=1}^{\infty} \frac{1}{n}$. Thus, if 


$$
\begin{aligned}
& \liminf _{n \rightarrow \infty} n^{p_{K}} v^{n}\left(A_{n+1}\right)>0, \\
& \limsup _{n \rightarrow \infty} n^{p_{K}} v^{n}\left(A_{n+1}\right)<\infty
\end{aligned}
$$

it follows that $\int_{\mathbf{M}^{2 \times 2}}|\lambda|^{p_{K}} d \nu(\lambda)=\infty$ and for every $p<p_{K}, \int_{\mathbf{M}^{2 \times 2}}|\lambda|^{p} d \nu(\lambda)<\infty$.

After plugging the value of $v^{n}\left(A_{n+1}\right)$ into (4.12) we are led to study the behavior of the sequence, $a_{n}=\prod_{i=1}^{n} \lambda_{i}$. The following basic manipulation show that it behaves like $n^{-p_{K}}$. Firstly we handle the product by using (4.7) and taking logarithms. This gives

$$
\left|\log \left(a_{n}\right)+\sum_{i=1}^{n} \frac{2 K}{(i+1)(K-1)}\right| \leqslant c(n),
$$

where $\sup _{n} c(n) \leqslant C \leqslant \infty$. Since $p_{K}=\frac{2 K}{K-1}$ and $\left|\log (n)-\sum_{i=1}^{n} \frac{1}{i+1}\right| \leqslant c_{0}$, we arrive to

$$
\left|\log \left(a_{n}\right)+p_{K} \log (n)\right| \leqslant c_{1}<\infty
$$

for every $n \in \mathbb{N}$.

Therefore, (4.12) is satisfied and the staircase laminate $v$ verifies (4.1).

Remark 4.2. - It is the fact that the auxiliary values $\left\{A_{n}\right\}$ are asymptotically close to the set $E_{1} \cap D=\{(a, a): a \in \mathbb{R}\}$ that characterizes the integrability of the measure $v$. Generally, let $\left\{\tilde{A_{n}}\right\} \subset \mathcal{Q}$ be a sequence of auxiliary matrices ordered as in (4.2) and such that

$$
\lim _{n \rightarrow \infty} \frac{\tilde{A}_{n}^{2}}{\tilde{A}_{n}^{1}}=t .
$$

Then it can be shown that if we perform the above scheme to obtain a laminate $\tilde{v}$, the threshold for the integrability of $\tilde{v}$ is equal to

$$
\frac{K}{(K-t)}+\frac{K}{(K-1 / t)}
$$

\section{Correcting sequences via Beltrami operators}

Consider the staircase-laminate $v$. By the construction it is a $p$-laminate in the sense of Definition 3.3 for every $1<p<p_{K}$. Hence by Theorem 3.4, there exists a sequence $\left\{f_{j}\right\} \in W^{1, p}\left(Q, \mathbb{R}^{2}\right)$ such that

$$
D f_{j \sharp}\left(\mathcal{L}_{R}^{n}\right) \stackrel{\star}{\rightarrow} v
$$

for every $R \Subset Q,|R|>0$, and $\left\{f_{j}\right\}$ is uniformly bounded in $W^{1, p}\left(Q, \mathbb{R}^{2}\right)$ for every $1<p<p_{K}$. In addition, the measure $v$ satisfies the requirements of Remark 3.3 and is supported on the set $E \backslash B(0, \varepsilon)$ for some $\varepsilon>0$. Thus,

$$
\lim _{j \rightarrow \infty} \mid\left\{z \in Q: D f_{j}(z) \notin E \text { or } D f_{j}(z)=0\right\} \mid=0 .
$$

The fact that $E$ is related to an elliptic equation allows us to "project" the sequence $\left\{f_{j}\right\}$ to another sequence $\left\{g_{j}\right\}$ such that $\left\{D g_{j}\right\}$ take values in $E$ and converges to $D f_{j}$ in $L^{p}$. 
PROPOSITION 5.1. - Let $v$ be the staircase laminate and $\left\{f_{j}\right\}$ the generating sequence with the properties (5.1) and (5.2). Let $k=(K-1) /(K+1)$. Then there exists a sequence of Beltrami coefficients $\mu_{j} \in L^{\infty}(Q,\{k,-k\})$ and a sequence $\left\{g_{j}\right\} \in$ $W^{1, p}\left(Q, \mathbb{R}^{m}\right)$ for all $2 \leqslant p<p_{K}$ such that:

$$
\bar{\partial} g_{j}(z)-\mu_{j}(z) \overline{\partial g_{j}}(z)=0
$$

for a.e.z in $Q$, and

$$
\lim _{j \rightarrow \infty}\left\|D f_{j}-D g_{j}\right\|_{L^{p}(Q)} \rightarrow 0 .
$$

Proof. - Declare $\Omega_{j}=\left\{z \in Q: D f_{j}(z) \in E\right.$ and $\left.D f_{j}(z) \neq 0\right\}$. Then (5.2) reads as

$$
\lim _{j \rightarrow \infty}\left|Q \backslash \Omega_{j}\right|=0 .
$$

Define

$$
\mu_{j}(z)= \begin{cases}\bar{\partial} f_{j}(z) / \overline{\partial f_{j}}(z) & \text { if } z \in \Omega_{j}, \\ k & \text { if } z \in Q \backslash \Omega_{j} .\end{cases}
$$

It is easy to see from the expression of the set $E$ in complex coordinates (2.2) that $\mu_{j} \in L^{\infty}(Q,\{k,-k\})$. The key point in the proof is that (5.5) implies that each $\left\{f_{j}\right\}$ satisfies a non homogeneous Beltrami equation with right hand side going to zero in $L^{p}$ for every $p<p_{K}$. The argument is the following: By the definition of $\mu_{j}$

$$
\bar{\partial} f_{j}(z)-\mu_{j}(z) \overline{\partial f_{j}}(z)=\left(\bar{\partial} f_{j}(z)-k \overline{\partial f_{j}(z)}\right) \chi_{Q \backslash \Omega_{j}}(z)
$$

in $Q$. Let $h_{j}(z)=\left(\bar{\partial} f_{j}(z)-k \overline{\partial f_{j}}(z)\right) \chi_{Q \backslash \Omega_{j}}(z)$ and consider exponents $2<p<p^{\prime}<p_{K}$. Then Hölder's inequality with exponents $p^{\prime} / p, p^{\prime} /\left(p^{\prime}-p\right)$ implies that $\int_{\mathbb{C}}\left|h_{j}\right|^{p} d z \leqslant$ $C\left\|D f_{j}\right\|_{L^{p^{\prime}}(Q)}\left|\Omega_{j}\right|^{\left(p^{\prime}-p\right) / p^{\prime}}$. By the definition of $v,\left\|D f_{j}\right\|_{p^{\prime}}$ is uniformly bounded and hence

$$
\lim _{j \rightarrow \infty} \int_{\mathbb{C}}\left|h_{j}\right|^{p} d z \rightarrow 0,
$$

for every $p<p_{K}$. Here after, the argument is similar to those in [5]. We sketch the proof, that goes in the same way that the proof of Theorem 1.2 in [5]. First we extend $\mu_{j}$ to the whole complex plane as

$$
\tilde{\mu}_{j}(z)= \begin{cases}\mu_{j}(z) & \text { if } z \in Q \\ 0 & \text { otherwise. }\end{cases}
$$

Now we use the two integral operators naturally related to the theory of quasiconformal mappings; The Cauchy transform $P$,

$$
P f(z)=\frac{-1}{\pi} \int_{\mathbb{C}} \frac{f(w)}{w-z} d x d y
$$


and the Beurling-Ahlfors transform $S$

$$
(S f)(z)=\frac{-1}{\pi} \int_{\mathbb{C}} \frac{f(w)}{(w-z)^{2}} d x d y .
$$

For definitions and proofs of their properties see [1]. These operators satisfy that for smooth compactly supported $h$,

$$
\bar{\partial} P(h)=h, \quad \partial P(h)=S(h) .
$$

Moreover both operators are continuous from $L^{p}(\mathbb{C})$ into itself. Therefore (5.9) extends to $L^{p}, p>1$, in the distributional sense.

Then if we consider the sequence $\left\{F_{j}\right\}$

$$
F_{j}=P\left(\left(I-\tilde{\mu}_{j} \bar{S}\right)^{-1}\left(h_{j}\right)\right)
$$

it is easy to see that $g_{j}=\left(F_{j}-f_{j}\right) \chi_{Q}$ satisfies the equation

$$
\bar{\partial} g_{j}-\mu_{j} \overline{\partial g_{j}}=0 \quad \text { in } Q .
$$

Furthermore, by (5.7), $\left\{h_{j}\right\}$ tends to zero in $L^{p}$. Recall that through the whole proof we are assuming that $2 \leqslant p<p_{K}$. Hence, by Theorem 3 in [4], $\left(I-\tilde{\mu}_{j} \bar{S}\right)^{-1}$ is a bounded operator from $L^{p}$ into itself [4]. It follows that $\left\{\left(I-\tilde{\mu}_{j} \bar{S}\right)^{-1} h_{j}\right\}$ tends to zero in $L^{p}(\mathbb{C})$ as well. This fact together with (5.9) and the boundness of $S$ imply that $\left\{D F_{j}\right\}$ also converges to zero in $L^{p}(\mathbb{C})$. We have proved that $\left\{g_{j}\right\}$ satisfy (5.3) and (5.4).

We will need that the convergence in $L^{p}$ implies that the limit of the distributional measures are the same, i.e. (5.4) implies that for every $R \Subset Q,|R|>0$

$$
\lim _{j \rightarrow \infty} D f_{j \sharp}\left(\mathcal{L}_{R}^{n}\right)=\lim _{j \rightarrow \infty} D g_{j_{\sharp}}\left(\mathcal{L}_{R}^{n}\right)
$$

in the weak star topology of $\mathcal{M}\left(\mathbf{M}^{2 \times 2}\right)$. Actually convergence in measure is enough for (5.11) to hold.

Remark 5.2. - The above proposition holds for every $W^{1, p}$-GYM supported in $E$ with $1+k<p$. In this case we do not know if (5.2) holds since a priory the generating sequence only converges to $E$ in measure. Therefore, a more subtle argument is needed to choose the Beltrami coefficients. One option is using the so-called Measurable Selection Lemma to find a projection of the generating sequence to $E$. Other is noticing that $E=F^{-1}(0)$ with $F(A)=\min \left\{\left|A_{\bar{z}}-k \overline{A_{z}}\right|^{p},\left|A_{\bar{z}}+k \overline{A_{z}}\right|^{p}\right\}$. Then the fact that $\int_{\mathbb{C}} F(\lambda) d \nu(\lambda)=0$ gives a choice of appropriate Beltrami coefficients.

\section{Proof of Theorem 1.1}

We start with the staircase-laminate $v$ constructed in Section 3. Firstly, by Theorem 3.4 applied to $v$ we obtain a generating sequence $\left\{f_{j}\right\}$. Then Proposition 5.1 provides a generating sequence $\left\{g_{j}\right\}$ and a sequence of Beltrami coefficients $\left\{\mu_{j}\right\} \in L^{\infty}(Q,\{k,-k\})$ 
such that

$$
\bar{\partial} g_{j}(z)-\mu_{j}(z) \overline{\partial g_{j}(z)}=0
$$

in $Q$. Next, let us recall that if $g_{j}(z)=u_{j}(z)+i v_{j}(z)$ for $u_{j}$ and $v_{j} \in W_{\text {loc }}^{1,2}(Q)$, it is an algebraic computation to show that

$$
\operatorname{div}\left(\rho_{j}(z) \nabla u_{j}(z)\right)=0
$$

in $Q$, where $\rho_{j}(z)=\frac{1-\mu_{j}(z)}{1+\mu_{j}(z)}$. For the sake of completeness we show the calculation. First we observe that

$$
2 \bar{\partial} g_{j}(z)=\nabla u_{j}(z)+J \nabla v_{j}(z), \quad 2 \overline{\partial g_{j}(z)}=\nabla u_{j}(z)-J \nabla v_{j}(z) .
$$

Thus, (6.1) becomes

$$
\nabla u_{j}(z)+J \nabla v_{j}(z)=\mu_{j}(z)\left(\nabla u_{j}(z)-J \nabla v_{j}(z)\right),
$$

a.e. $z$ in $Q$. After rearranging this equation we obtain

$$
\frac{1-\mu_{j}(z)}{1+\mu_{j}(z)} \nabla u_{j}(z)=-J \nabla v_{j}(z)
$$

and (6.2) follows by recalling that $J$ sends curl free vector fields to divergence free vector fields. Now, when $\mu_{j}(z)=k, \rho_{j}(z)=(1-k) /(1+k)=1 / K$ and when $\mu_{j}(z)=$ $-k, \rho_{j}(z)=(1+k) /(1-k)=K$, so (6.2) is an elliptic isotropic equation like (1.1). Moreover, the bounds on the $\rho_{j}$ and (6.3) imply that for every $1 \leqslant p<\infty$

$$
\int_{R}\left|\nabla u_{j}(z)\right|^{p} d z \leqslant \int_{R}\left|D g_{j}(z)\right|^{p} d z \leqslant C(K) \int_{R}\left|\nabla u_{j}(z)\right|^{p} d z .
$$

To conclude we use the following basic consequence of the Monotone Convergence Theorem.

Lemma 6.1 [7, Proposition 2.15]. - Let $v_{j} \stackrel{\star}{\rightarrow} v$ in $\mathcal{M}\left(\mathbf{M}^{2 \times 2}\right)$ and $f$ be a positive continuous function on $\mathbf{M}^{2 \times 2}$. Then,

$$
\int_{\mathbf{M}^{2 \times 2}} f(\lambda) d v(\lambda) \leqslant \lim \inf _{j \rightarrow \infty} \int_{\mathbf{M}^{2 \times 2}} f(\lambda) d v_{j}(\lambda) .
$$

Take $v$ equal to the staircase laminate, $v_{j}=D g_{j_{\sharp}}\left(\mathcal{L}_{R}^{n}\right)$ and $f(\lambda)=|\lambda|^{p_{K}}$ in the lemma. The left hand side is equal to $\infty$ by (4.1) which yields that

$$
\lim \inf _{j \rightarrow \infty} \frac{1}{|R|} \int_{R}\left|D g_{j}\right|^{p_{K}}=\infty
$$


and, by (6.4), that

$$
\lim \inf _{j \rightarrow \infty} \int_{R}\left|\nabla u_{j}\right|^{p_{K}}=\infty .
$$

Moreover, since $\left\{D g_{j}\right\}$ is uniformly bounded in $L^{p}(Q)$ for every $p<p_{K}(6.4)$ implies, after normalization, that

$$
\left\|u_{j}(z)\right\|_{W^{1,2}(Q, \mathbb{R})} \leqslant 1 .
$$

Therefore, $\rho_{j}(z)=\frac{1-\mu_{j}(z)}{1+\mu_{j}(z)}$ and $u_{j}(z)$ equal to the real part of $g_{j}(z)$ prove Theorem 1.1.

Proof of the Corollary 1.2. - Consider a union of disjoint balls $\left\{B_{i}\left(a_{i}, r_{i}\right)\right\}_{i=1}^{\infty} \Subset Q$. Let $\eta \in C_{0}^{\infty}(B(0,1))$ be a cut-off function such that $\eta(z)=1$ if $|z| \leqslant 1 / 2$. For every $i$ let $T_{i}(z)=\left(z-a_{i}\right) / r_{i}$, be a similarity satisfying $T_{i}\left(B_{i}\right)=B(0,1)$. Define then, $\eta_{i}=$ $r_{i} \eta\left(T_{i}(z)\right) \in C_{0}^{\infty}\left(B_{i}\right)$. Use now (6.5) with $R=B\left(a_{i}, r_{i} / 2\right)$ to select $j(i)$ such that

$$
\int_{B\left(a_{i}, r_{i} / 2\right)}\left|D g_{j(i)}(z)\right|^{p_{K}} d z \geqslant \frac{1}{r_{i}} .
$$

Setting $j(i)=i$ gives sequences $\left\{g_{i}\right\}_{i=1}^{\infty},\left\{\mu_{i}\right\}_{i=1}^{\infty}$. Let

$$
\mu=\sum_{i=1}^{\infty} \mu_{i} \chi_{B_{i}}+k \chi_{Q \backslash \bigcup_{i=1}^{\infty} B_{i}}
$$

and

$$
g=\sum_{i=1}^{\infty} g_{i} \eta_{i}
$$

Then we have that

$$
\bar{\partial} g(z)-\mu \overline{\partial g}(z)=F
$$

in $Q$.

Explicitly the vector field $F$ is given by

$$
F=\sum_{i=1}^{\infty}\left(\bar{\partial} \eta_{i}(z)-\mu_{i} \overline{\partial \eta_{i}}(z)\right) g_{i} .
$$

Thus, we can use the Sobolev embedding for the $g_{i}$ and that $\left\|\nabla \eta_{i}\right\|_{\infty}=\|\nabla \eta\|_{\infty}$ to see that $F \in L^{\infty}(Q)$. Regarding the integrability of $|D g|^{p_{K}}$ we have that

$$
\int_{Q}|D g|^{p_{K}} d z \geqslant \sum_{i=1}^{\infty} r_{i} \int_{B\left(a_{i}, r_{i} / 2\right)}\left|D g_{i}\right|^{p_{K}},
$$

which, after plugging (6.6), implies that

$$
\int_{Q}|D g(z)|^{p_{K}} d z=\infty .
$$


Finally, the same calculation that in the homogeneous case (6.1)-(6.3) shows that $u=\operatorname{Re}(g)$ satisfies the equation

$$
\operatorname{div}(\rho \nabla u)=\operatorname{div} \frac{1}{1+\mu(z)} 2 F,
$$

where $\rho(z)=\frac{1-\mu(z)}{1+\mu(z)}$. This together with (6.10) proves the corollary.

Remark 6.2. - From the viewpoint of physics, the exponent $p_{K}$ being low means that there areas where the concentration of the electric field is quantitatively high. In [18] is proposed to investigate whether there are areas where the electric field is especially feeble. For this question we search for the largest exponent $q_{K}$ such that for every $q<q_{K}$

$$
\int_{R}|\nabla u(z)|^{-q} d z<C(R, p),
$$

where $u$ is a solution to (1.1). To avoid technical problems with the singular set we assume that the quasiregular mapping $f$ such that

$$
D f=\left(\begin{array}{c}
\nabla u \\
J \rho \nabla u
\end{array}\right)
$$

is a local homeomorphism everywhere. This for example is guaranteed if we assume affine boundary values for $u$ (see [15]).

Essentially the same example shows that $q_{K}=2 /(K-1)$. We consider the staircase laminate $v$ and a generating sequence $\left\{f_{j}\right\}$. We define the same sequence of Beltrami coefficients $\left\{\tilde{\mu}_{j}\right\}$ as in (5.8). The difference is that this time we correct the sequence $f_{j}$ with the Beltrami operator $\left(I-\tilde{\mu_{j}} S\right)^{-1}$ to obtain a sequence $\left\{g_{j}\right\}$ of solutions of

$$
\bar{\partial} g_{j}(z)-\tilde{\mu}_{j}(z) \partial g_{j}(z)=0
$$

in $Q$. Observe that we are not taking the conjugate of $\partial g_{j}(z)$ so $\tilde{\mu}_{j}$ are standard complex dilatations [1]. Assume for a moment that the functions $g_{j}$ are injective. Then, the composition rule for Beltrami coefficients [1] shows that the functions $g_{j}^{-1}$ satisfy the equations

$$
\bar{\partial} g_{j}^{-1}(z)+\tilde{\mu}_{j}\left(g_{j}^{-1}(z)\right) \overline{\partial g_{j}^{-1}(z)}=0
$$

in $g_{j}(Q)$.

Therefore, by the discussion in Section 6, the real parts of $g_{j}^{-1}$ satisfy an isotropic equation like (1.1). Now, a change of variables gives that

$$
\int_{g_{j}(R)} J_{g_{j}^{-1}}(z)^{-1 /(K-1)} d z=\int_{R} J_{g_{j}}(w)^{K /(K-1)} d w
$$

for every $R$ compactly contained in $Q$. Notice that at this point one has to be careful because the domains $g_{j}(Q)$ are not the same. However, by the uniform quasisymmetry 
of $g_{j}$ we can find a domain $\tilde{Q}$, such that $\left\{g_{j}(Q)\right\}$ converges in the Hausdorff metric to $\tilde{Q}$. In turn this observation yields the result. If the $g_{j}$ are not injective (and they need no to be) we have to use [5, Theorem 1.5]. In our setting that result implies that there exists another sequence $\left\{F_{j}\right\}$ of injective solutions of (6.11) which generate the staircase laminate $v$ and hence, whose $p_{K}$-norm blows up. It follows that the sequence $\left\{\operatorname{Re}\left(F_{j}^{-1} \mid \tilde{Q}\right)\right\}$ prove that $q_{K} \leqslant 2 /(K-1)$. The other inequality follows from $[2,15]$.

Remark 6.3. - In the sequence we have obtained we have no control in the boundary values. This can be fixed in the following way. Firstly, it is clear that the generating sequence $\left\{f_{j}\right\}$ for the staircase laminate can be assumed to have affine boundary values. Then once we have our choice of Beltrami coefficients $\left\{\mu_{j}\right\}$ we can associate to them the right scalars $\left\{\rho_{j}\right\}$ as above. Then we look at the following boundary value problem

$$
\begin{aligned}
& \operatorname{div}\left(\rho_{j} \nabla u_{j}\right)=0, \\
& u_{j}-\operatorname{Re}\left(f_{j}\right) \in W_{0}^{1,2}(Q) .
\end{aligned}
$$

Then it can be shown (but it is a lengthier computation that the one we have presented) that since $\left\{\operatorname{Re}\left(f_{j}\right)\right\}$ solves an isotropic equation with right hand side going to zero $\left\|\nabla u_{j}-\nabla\left(\operatorname{Re}\left(f_{j}\right)\right)\right\|_{L^{2}} \rightarrow 0$. It is not hard to see that this is enough to guarantee that $\lim _{j \rightarrow \infty} \int_{R}\left|\nabla u_{j}\right|^{p_{K}} d z=\infty$ where $R$ is a compact set with positive measure.

\section{Acknowledgements}

I wish to thank countless stimulating conversations and ideas from Kari Astala. I also thank Marta Llorente, Vincenzo Nesi and Carlo Sbordone for their valuable comments and suggestions on the problem as well as for carefully reading preliminary versions of the manuscript.

\section{REFERENCES}

[1] L.V. Ahlfors, Lectures on Quasiconformal Mappings, Van Nostrand, Princenton, 1966.

[2] K. Astala, Area distortion of quasiconformal mappings, Acta Math. 173 (1994) 37-60.

[3] K. Astala, In preparation.

[4] K. Astala, T. Iwaniec, E. Saksman, Beltrami operators in the plane, Duke Math. J. 107 (1) (2001) 27-56.

[5] K. Astala, D. Faraco, Quasiregular mappings and Young measures, Proc. Roy. Soc. Edinburgh Sect. A, to appear.

[6] K. Astala, V. Nesi, Composites and quasiconformal mappings: New optimal bounds in two dimensions, Calc. Var. Partial Differential Equations, to appear.

[7] A. Braides, A. Defranceschi, Homogenization of Multiple Integrals, in: Oxford Lecture Series in Mathematics and its Applications, Vol. 12, Clarendon Press/Oxford University Press, New York, 1998.

[8] B.V. Bojarski, Generalized solutions of a system of differential equations of first order and of elliptic type with discontinuous coefficients, Mat. Sb. NS 43 (85) (1957) 451-503 (Russian).

[9] B. Dacorogna, P. Marcellini, Implicit Partial Differential Equations, in: Progress in Nonlinear Differential Equations and Their Applications, Vol. 37, Birkhäuser, 1999. 
[10] A. Erëmenko, D.H. Hamilton, On the area distortion by quasiconformal mappings, Proc. Amer. Math. Soc. 123 (9) (1995) 2793-2797.

[11] D. Faraco, Tartar Conjecture and Beltrami Operators, Preprint at the University of Helsinki, 2002.

[12] T. Iwaniec, C. Sbordone, Quasiharmonic fields, Ann. Inst. H. Poincaré Anal. Non Linaire 18 (5) (2001) 519-572.

[13] B. Kirheim, Geometry and rigidity of microstructures, Habilitation Thesis, Leipzig, 2001.

[14] P. Koskela, The degree of regularity of a quasiconformal mapping, Proc. Amer. Math. Soc. 122 (3) (1994) 769-772.

[15] F. Leonetti, V. Nesi, Quasiconformal solutions to certain first order systems and the proof of a conjecture of G.W. Milton, J. Math. Pures. Appl. (9) 76 (1997) 109-124.

[16] A. Marino, S. Spagnolo, Un tipo di approssimazione dell'operatore $\sum_{1}^{n} i j D_{i}\left(a_{i j}(x) D_{j}\right)$ con operatori $\sum_{1}^{n} j D_{j}\left(\beta(x) D_{j}\right)$, Ann. Scuola Norm. Sup. Pisa (3) 23 (1969) 657-673 (Italian).

[17] N.G. Meyers, An $L^{p}$ estimate for the gradient of solutions of second order elliptic divergence equations, Ann. Scuola Norm. Sup. Pisa (3) 17 (1963) 189-206.

[18] G. Milton, Modelling the properties of composites by laminates, in: Homogenization and Effective Moduli of Materials and Media, in: Ericksen, Kiderleher, Kohn, Lions (Eds.), IMA Volumes in Mathematics and its Applications, Vol. 1, Springer-Verlag, New York, 1986.

[19] C.B. Morrey, On the solution of quasi-linear elliptic partial differential equations, Trans. Amer. Math. Soc. 43 (1938) 126-166.

[20] S. Müller, V. Šverák, Unexpected solutions of first and second order partial differential equations, Doc. Math. J. DMV ICM (1998) 691-702.

[21] S. Müller, V. Šverák, Convex integrations with constrains and applications to phase transitions and partial differtential equations, MPI MIS, Preprint 98, 1999.

[22] P. Pedregal, Laminates and microstructure, Eur. J. Appl. Math. 4 (1993) 121-149.

[23] P. Pedregal, Parametrized Measures and Variational Principles, Birkhäuser, 1997.

[24] L.C. Piccinini, S. Spagnolo, On the Hölder continuity of solutions of second order elliptic equations in two variables, Ann. Scuola Norm. Sup. Pisa (3) 26 (1972) 391-402. 